\title{
ARTICLE
}

Clinical Research

\section{Prostate cancer prognosis after initiation of androgen deprivation therapy among statin users. A population-based cohort study}

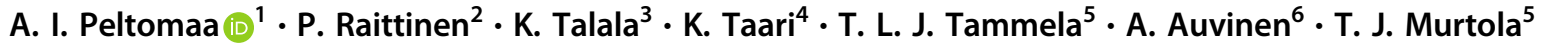

Received: 31 August 2020 / Revised: 7 February 2021 / Accepted: 11 March 2021 / Published online: 31 March 2021

(c) The Author(s) 2021. This article is published with open access

\begin{abstract}
Purpose Statins' cholesterol-lowering efficacy is well-known. Recent epidemiological studies have found that inhibition of cholesterol synthesis may have beneficial effects on prostate cancer (PCa) patients, especially patients treated with androgen deprivation therapy (ADT). We evaluated statins' effect on prostate cancer prognosis among patients treated with ADT.

Materials and methods Our study population consisted of 8253 PCa patients detected among the study population of the Finnish randomized study of screening for prostate cancer. These were limited to 4428 men who initiated ADT during the follow-up. Cox proportional regression model adjusted for tumor clinical characteristics and comorbidities was used to estimate hazard ratios for risk of PSA relapse after ADT initiation and prostate cancer death.

Results During the median follow-up of 6.3 years after the ADT initiation, there were 834 PCa deaths and 1565 PSA relapses in a study cohort. Statin use after ADT was associated with a decreased risk of PSA relapse (HR 0.73, 95\% CI 0.65-0.82) and prostate cancer death (HR 0.82; 95\% CI 0.69-0.96). In contrast, statin use defined with a one-year lag (HR $0.89,95 \%$ CI 0.76-1.04), statin use before ADT initiation (HR 1.12, 95\% CI 0.96-1.31), and use in the first year on ADT (HR 1.02, 95\% CI 0.85-1.24) were not associated with prostate cancer death, without dose dependency.

Conclusion Statin use after initiation of ADT, but not before, was associated with improved prostate cancer prognosis.
\end{abstract}

These authors contributed equally: A. Auvinen, T. J. Murtola

Supplementary information The online version contains supplementary material available at https://doi.org/10.1038/s41391021-00351-2.

A. I. Peltomaa antti.peltomaa@tuni.fi

1 University of Tampere, School of Medicine, Tampere, Finland

2 Department of Mathematics and Systems Analysis, Aalto University, School of Science, Espoo, Finland

3 Finnish Cancer Registry, Helsinki, Finland

4 Department of Urology, University of Helsinki and Helsinki University Hospital, Helsinki, Finland

5 Department of Urology, Tampere University Hospital, Tampere, Finland

6 University of Tampere, School of Health Sciences, Tampere, Finland

\section{Introduction}

Statins reduce blood cholesterol levels, which play a central role in androgen biosynthesis. Statins also limit cancer cell growth through the inhibition of the mevalonate pathway [1] and may inhibit lipogenesis which is important for cancer cells [2]. Furthermore, inhibition of acetyl-CoA modifies immune response. The immune response against tumors provides another possible mechanism for statins' anti-cancer effects [3]. Recent studies have concentrated on evaluating statins' efficacy in the treatment of cancer patients [1, 4-7].

In prostate cancer, statin use has been associated with a longer time to disease progression after the primary therapy $[1,4,5]$ and reduced disease-specific mortality [6]. Intraprostatic cholesterol metabolism and its upregulation have a key role in the development of castration-resistance during androgen deprivation therapy (ADT) [7]. Concordantly, the survival benefit associated with statin use may be strongest among men treated with ADT. However, there are only a few studies assessing statins' effect specifically in relation to ADT. Therefore, we evaluated the risk of prostate cancer death after ADT initiation with a special focus on the timing of statin use in relation to ADT. 
Fig. 1 Longitudinal design of the study. Initiation of androgen deprivation therapy (ADT) was defined to calculate statin use occuring before and after ADT. *Death/PSA relapse, emigration, or common closing date 1 January 2016.

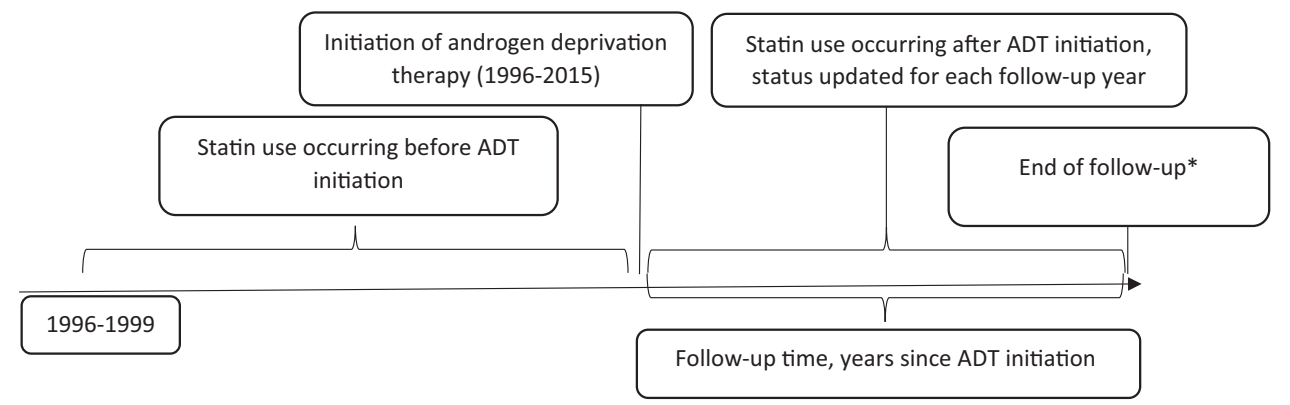

\section{Methods}

\section{Study cohort}

Finnish randomized study of screening for prostate cancer [8] is a randomized population-based trial assessing effects of systematic screening with prostate-specific antigen (PSA) on prostate cancer mortality. At baseline, 80458 men aged 55-67 years residing in the metropolitan areas of Helsinki or Tampere were randomized in 1996-1999 either to be invited for PSA-screening every 4 years or to the control arm without any intervention. The study population was identified from the population register center and linked to the comprehensive Finnish cancer registry (FCR) to exclude all prevalent prostate cancer cases. Incident PCa cases after baseline were identified from patient files and FCR. During 1996-2015 a total of 8253 men in the study population were diagnosed with prostate cancer, with 4428 initiating ADT after diagnosis. These men formed the study cohort for the present study. The longitudinal study design is graphically formatted in Fig. 1.

Information on TNM stage, PSA values (all measurements during 1996-2016), and Gleason score at diagnosis and possible radiation therapy (as primary or adjuvant/salvage treatment) were retrieved from hospital records and laboratory data of Fimlab or HUSLAB, main laboratory services providers in Tampere and Helsinki areas. By combining these tumor characteristics, we were able to categorize patients into prognostic risk groups for biochemical recurrence as defined by European Urological Association [9]. PCa patients with PSA $<10$ at diagnosis, localized disease, T1-2a, and Gleason score 6 or lower were classified as low-risk cases. Information on BMI was available for 805 men at the time of screening. PCa cases with PSA above 20, Gleason score above 7, locally advanced disease, $\mathrm{T} 2 \mathrm{c}-4$ or $\mathrm{N}+$ were classified as high-risk cancers. All de novo metastatic (M1) cases were also categorized as high risk. All other PCa cases were grouped into intermediate-risk groups. PSA relapse was defined as two consecutive rises of at least $50 \%$ from nadir PSA provided that the final PSA was over 2.
Statistics Finland registers all deaths occurring in Finland. In this analysis deaths with prostate cancer (ICD-10 code C61) as the primary cause of death were defined as prostate cancer deaths. Statistics Finland has also approved (TK-53-1330-18) our research permission.

\section{Information on medication use}

The study cohort was linked to the National prescription database maintained by the social insurance institute (SII) of Finland. SII provides reimbursements for purchases of physician-prescribed medication in Finland. The Finnish reimbursement system has been described in detail previously [10]. ADT use was defined as any purchase of GnRH agonists, GnRH antagonists, or antiandrogens during 1995-2015, as identified by drug-specific ATC codes. Similarly, information on all statins, as well as, antidiabetic drugs, antihypertensive drugs, and non-steroidal antiinflammatory drugs were obtained.

To complement our data on ADT, information on surgical bilateral orchiectomies performed during 1995-2015 were obtained from the care register for health care (HILMO) maintained by the National Institute for Health and Welfare. Orchiectomies were identified using the nordic classification of procedures code KFC10.

The initiation of ADT use was defined as the date of surgical orchiectomy or the first reimbursement for prescription of any ADT medication, whichever occurred first.

The annual cumulative mg amount of use for each statin during follow-up was calculated by adding together all purchases within a given calendar year. Amount of use was standardized between different statins by dividing the annual total $\mathrm{mg}$ amount of purchases with the amount corresponding to a defined daily dose (DDD) as listed by the World Health Organization [11]. Each year with any recorded statin purchases was considered as a year of usage. Cumulative statin DDDs and years of usage were calculated by adding together yearly purchases. Average yearly DDDs, i.e., the intensity of statin use was calculated by dividing yearly cumulative DDDs with a cumulative number of years of usage. 
Table 1 Population characteristics, the cohort of prostate cancer patients treated with androgen deprivation therapy.

\begin{tabular}{|c|c|c|}
\hline & \multicolumn{2}{|c|}{ Participants starting ADT } \\
\hline & \multicolumn{2}{|c|}{$\begin{array}{l}\text { Statin use during the follow-up } \\
(1996-2015)\end{array}$} \\
\hline & None & Any \\
\hline No of men & 1884 & 2544 \\
\hline No of PCa deaths & $482(25.6 \%)$ & $352(13.8 \%)$ \\
\hline No of overall deaths & $1009(53.6 \%)$ & $918(36.1 \%)$ \\
\hline $\begin{array}{l}\text { Median (IQR) follow-up time (years) } \\
\text { after ADT initiation }\end{array}$ & $5.5(2.6-9.4)$ & $6.9(3.6-10.6)$ \\
\hline Mean age at PCa diagnosis (years) & 69.0 & 69.7 \\
\hline Mean age at ADT initiation & 70.1 & 70.9 \\
\hline BMI; median (IQR) & $26.0(23.7-28.7)$ & $26.8(24.7-29.1)$ \\
\hline \multicolumn{3}{|l|}{ Tumor stage at diagnosis } \\
\hline $\mathrm{T} 1-2$ & $1146(60.8 \%)$ & $1781(70.0 \%)$ \\
\hline T3-4 & $737(39.2 \%)$ & $763(30.0 \%)$ \\
\hline Unknown & $1(0.02 \%)$ & 0 \\
\hline \multicolumn{3}{|l|}{ Tumor Gleason grade } \\
\hline 6 or lower & $583(30.9 \%)$ & $886(34.8 \%)$ \\
\hline 7 & $671(35.6 \%)$ & $957(37.6 \%)$ \\
\hline $8-10$ & $575(30.5 \%)$ & $660(25.9 \%)$ \\
\hline Metastatic disease at diagnosis (M1) & $316(16.8 \%)$ & $240(9.4 \%)$ \\
\hline \multicolumn{3}{|l|}{ PSA level at diagnosis } \\
\hline 20 or less & $1076(57.1 \%)$ & $1665(65.4 \%)$ \\
\hline Above 20 & $645(34.2 \%)$ & $640(25.2 \%)$ \\
\hline Unknown & $163(8.7 \%)$ & $239(9.4 \%)$ \\
\hline \multicolumn{3}{|l|}{ Choice of primary treatment } \\
\hline Active surveillance & $142(7.5 \%)$ & $248(9.7 \%)$ \\
\hline Radical prostatectomy & $148(7.9 \%)$ & $184(7.2 \%)$ \\
\hline Radical radiotherapy & $205(10.9 \%)$ & $333(13.1 \%)$ \\
\hline LHRH & $986(52.3 \%)$ & $1434(56.4 \%)$ \\
\hline Other & $403(21.4 \%)$ & $345(13.6 \%)$ \\
\hline PSA relapse & $723(38.4 \%)$ & $842(33.1 \%)$ \\
\hline \multicolumn{3}{|l|}{ EAU risk group } \\
\hline Low-risk & $288(15.3 \%)$ & $480(18.9 \%)$ \\
\hline Intermediate-risk & $625(33.2 \%)$ & $1014(39.9 \%)$ \\
\hline High-risk & $971(51.5 \%)$ & $1050(41.3 \%)$ \\
\hline \multicolumn{3}{|l|}{ Use of other medication } \\
\hline Andiabetic drugs & $220(11.7 \%)$ & $745(29.3 \%)$ \\
\hline Antihypertensive drugs & $1152(61.1 \%)$ & $2173(85.4 \%)$ \\
\hline NSAIDs & $1545(82.0 \%)$ & $2218(87.2 \%)$ \\
\hline Aspirin & $133(7.1 \%)$ & $448(17.6 \%)$ \\
\hline \multicolumn{3}{|c|}{ Type of ADT (categories not mutually exclusive) } \\
\hline GnRH agonist/antagonist & $1497(79.5 \%)$ & $2096(82.4 \%)$ \\
\hline Antiandrogens & $1116(59.2 \%)$ & $1390(54.6 \%)$ \\
\hline Orchiectomy & $210(11.1 \%)$ & $184(7.2 \%)$ \\
\hline \multicolumn{3}{|l|}{ Radiation therapy } \\
\hline None & $1009(53.6 \%)$ & $1111(43.7 \%)$ \\
\hline Yes & $875(46.4 \%)$ & $1433(56.3 \%)$ \\
\hline \multicolumn{3}{|l|}{ Socioeconomic status } \\
\hline Employed & $234(12.4 \%)$ & $269(10.6 \%)$ \\
\hline Unemployed & $64(3.4 \%)$ & $47(1.8 \%)$ \\
\hline Retired & $1562(82.9 \%)$ & $2210(86.9 \%)$ \\
\hline Unknown & $24(1.3 \%)$ & $18(0.7 \%)$ \\
\hline \multicolumn{3}{|l|}{ Marital status } \\
\hline Single/divorced/widow & $626(33.2 \%)$ & $618(24.3 \%)$ \\
\hline Married/registered partnership & $1258(66.8 \%)$ & $1926(75.7 \%)$ \\
\hline
\end{tabular}

\section{Statistical analysis}

Cox proportional hazards model was used to estimate hazard ratios (HRs) and 95\% confidence intervals (95\% CIs) for risk of PSA relapse, prostate cancer death, and death due to any cause after initiation of ADT. Follow-up time for these analyses started at ADT initiation and ended in PSA relapse or death, emigration, or common closing date 1 January 2016, whichever came first. We used model adjustment for randomization group, age, tumor risk group, simultaneous use of NSAIDs, antidiabetics, or antihypertensive drugs, and whether the participant received radiation therapy in addition to ADT.

Statin use after ADT initiation was analyzed as a timedependent variable; status of usage, as well as the cumulative amount, duration, and intensity of use, were updated separately for each follow-up year based on recorded statin purchases. Statin use before ADT initiation was analyzed as the time-fixed variable. Usage status of all other medications (NSAIDs, antidiabetics, and antihypertensive drugs) was not allowed to change on a yearly basis, and participants were regarded as a user if a person had one reimbursed purchase during the follow-up.

To estimate the latency of the risk associations with statin use we performed lag time analyses using statin exposure occurring 1-3 years earlier instead of the contemporary exposure, e.g., for outcomes occurring on the fifth follow-up year we used statin use from the fourth year of follow-up as the exposure in the 1-year lag time analysis.

\section{Results}

\section{Population characteristics}

Of the 4428 ADT-treated patients, 2544 (47.9\%) had used statins during the follow-up. During the median follow-up of 6.3 years from the initiation of ADT, there were 482 and 352 deaths due to prostate cancer and 723 and 842 PSA relapses among nonusers and statin users, respectively (Table 1). ADT method among statin users was slightly more often GnRH agonists/antagonists and less often orchiectomy.

\section{Risk of prostate cancer death by statin use before ADT initiation}

Statin use before ADT initiation was not associated with prostate cancer-specific survival (HR 1.12; 0.95 CI $0.96-1.31$ ) (Table 2). No dose-dependence by yearly dosing was observed, either. 
Table 2 Risk of prostate cancer death by statin use before ADT in a cohort of prostate cancer patients treated with ADT.

\begin{tabular}{llll}
\hline & \multicolumn{3}{l}{ Risk of PCa death } \\
\hline Statin use before ADT & $\begin{array}{l}\text { No of participants/ } \\
\text { PCa deaths } \\
\text { None }\end{array}$ & Age-adjusted & Multivariable adjusted* \\
Any & $1504 / 593$ & Reference & Reference \\
Intensity of statin use & & $0.97(0.84-1.13)$ & $1.12(0.96-1.31)$ \\
First tertile (below 120 DDD/year) & $508 / 82$ & $0.90(0.71-1.13)$ & $1.09(0.86-1.38)$ \\
Second tertile (120-200 DDD/year) & $510 / 90$ & $1.07(0.86-1.34)$ & $1.19(0.95-1.50)$ \\
Third tertile (above 200 DDD/year) & $506 / 69$ & $0.94(0.74-1.22)$ & $1.07(0.83-1.38)$ \\
\hline
\end{tabular}

*Calculated using Cox regression with adjustment for age, tumor risk group, randomization group, use of other medication (antidiabetic and antihypertensive drugs, NSAIDs), and whether participants received radiation therapy in addition to ADT.

\section{Risk of PSA relapse after ADT initiation by statin use}

Statin use after ADT initiation was linked to a decreased risk of PSA relapse (HR 0.73; 0.95 CI 0.65-0.82) when adjusted by age, randomization group, medications, and $\mathrm{PCa}$ risk group. In lag-time analysis, risk decrease remained statistically significant in the 1-year lag-time analysis.

\section{Risk of prostate cancer death by statin use after ADT initiation}

In age-adjusted and multivariable-adjusted analyses, statin use after ADT was associated with a decreased risk of prostate cancer death (HR 0.82; 0.95 CI 0.69-0.96). Median PCa survival times after ADT initiation were 6.8 and 5.9 years for statin users and non-users, respectively. The association was dose-dependent and was observed most clearly in high-intensity statin use (HR 0.58 ; $0.95 \mathrm{CI}$ $0.44-0.76$ ) for men who had used at least $210 \mathrm{DDD} /$ year. No significant risk decrease was observed in the lowest intensity tertile (HR 0.94; 0.95 CI 0.69-1.29) for men who had used 92 DDD/year or less (Table 3). Similar decreasing risk trends were observed also by cumulative DDD amount and years of statin use (Supplementary Table 1).

In the lag-time analysis, the risk decrease was slightly attenuated and no statistically significant difference was observed (Table 3 ).

\section{Subgroup analyses}

No statistically significant effect modification was observed by FinRSPC study arm, PCa risk group, use of antidiabetic drugs, or use of radiation therapy in addition to ADT in stratified analyses (Table 4 and Fig. 2). Statin use after ADT initiation was associated with decreased mortality also in almost all subgroups.

\section{Risk of death (all-cause mortality) by statin use before and after ADT initiation}

Statin use after ADT initiation, but not before, was associated with decreased all-cause mortality (HR $0.84 ; 0.95 \mathrm{CI}$ 0.76-0.93) (Table 5). The risk decrease was dose-dependent and was observed most clearly in high-intensity statin users.

\section{Sensitivity analyses}

To estimate whether risk estimates among statin users were affected by presumably increased cardiovascular mortality among statin users, we performed Fine and Gray competing risks regression analysis with death due to cardiovascular disease (ICD-10 codes I20-I25) as the competing outcome. In this analysis statin use before ADT was associated with a slightly elevated risk of PCa death (HR 1.17, 95\% CI 0.99-1.38) whereas statin use after ADT continued to be associated with lowered risk (HR $0.68,95 \%$ CI $0.58-0.80$ ). Therefore lowered risk of $\mathrm{PCa}$ death is unlikely to be explained by a concomitantly elevated risk of cardiovascular death.

To assess how statin use at the time of ADT initiation may associate with PCa survival, we performed analysis including only statin use at baseline, i.e., the first year of ADT use, with follow-up starting at the second year of follow-up. In this analysis, statin use was not associated with PCa death (HR 1.02, 95\% CI 0.85-1.24) confirming that statin users at baseline are at equal risk of dying of $\mathrm{PCa}$ compared to non-users, with no bias to favor statin users.

\section{Discussion}

We have shown in a cohort of FinRSPC PCa patients that statin use after initiation of ADT, but not before, is associated with improved prostate cancer survival. The risk 
Table 3 Risk of prostate cancer death and PSA relapse by statin use after ADT initiation in a cohort of prostate cancer patients treated with ADT.

\begin{tabular}{|c|c|c|c|c|c|}
\hline & \multirow[b]{2}{*}{ No of participants/deaths } & \multicolumn{4}{|c|}{ Risk of prostate cancer death } \\
\hline & & Age-adjusted & Multivariable adjusted & 1-year lag-time & 3-year lag-time \\
\hline Statin use after ADT & & $\mathrm{HR}(95 \% \mathrm{CI})$ & $\mathrm{HR}(95 \% \mathrm{CI})^{*}$ & $\mathrm{HR}(95 \% \mathrm{CI})^{*}$ & $\mathrm{HR}(95 \% \mathrm{CI})^{*}$ \\
\hline None & $2707 / 582$ & Reference & Reference & Reference & Reference \\
\hline Any & $1721 / 252$ & $0.68(0.59-0.80)$ & $0.82(0.69-0.96)$ & $0.89(0.76-1.04)$ & $0.90(0.77-1.06)$ \\
\hline \multicolumn{6}{|l|}{ Intensity of statin use (DDDs/year) } \\
\hline First tertile (below 92 DDD/year) & $574 / 160$ & $0.83(0.61-1.14)$ & $0.94(0.69-1.29)$ & $0.99(0.71-1.37)$ & $0.86(0.58-1.29)$ \\
\hline Second tertile (92-210 DDD/year) & $572 / 70$ & $0.60(0.47-0.75)$ & $0.67(0.53-0.84)$ & $0.73(0.57-0.93)$ & $0.87(0.67-1.15)$ \\
\hline Third tertile (above 210 DDD/year) & $575 / 22$ & $0.48(0.37-0.63)$ & $0.58(0.44-0.76)$ & $0.88(0.69-1.14)$ & $0.93(0.68-1.26)$ \\
\hline \multicolumn{6}{|c|}{ Risk of PSA relapse } \\
\hline Statin use after ADT & No of participants/PSA relapses & Age-adjusted & Multivariable adjusted* & 1-year lag-time & 3-year lag-time \\
\hline None & $2707 / 957$ & Reference & Reference & Reference & Reference \\
\hline Any & $1721 / 608$ & $0.65(0.58-0.72)$ & $0.73(0.65-0.82)$ & $0.85(0.76-0.95)$ & $0.97(0.86-1.09)$ \\
\hline
\end{tabular}

*Calculated using Cox regression with adjustment for age, tumor risk group, randomization group, use of other medication (antidiabetic and antihypertensive drugs, NSAIDs), and whether participants received radiation therapy in addition to ADT.

Table 4 Risk of prostate cancer death by statin use after ADT stratified by various baseline variables.

\begin{tabular}{|c|c|c|c|c|}
\hline & & \multirow[t]{2}{*}{ Participants/deaths } & \multicolumn{2}{|c|}{$\begin{array}{l}\text { Risk of PCA death among ADT treated patients } \\
\text { with statin use }\end{array}$} \\
\hline & & & $\begin{array}{l}\text { Age-adjusted } \\
\text { HR ( } 95 \% \text { CI) }\end{array}$ & $\begin{array}{l}\text { Multivariable adjusted } \\
\text { HR }(95 \% \mathrm{CI})^{*}\end{array}$ \\
\hline \multirow[t]{2}{*}{ FinRSPC randomization group } & Control arm & $2815 / 547$ & $0.70(0.58-0.84)$ & $0.80(0.66-0.98)$ \\
\hline & Screening arm & $1613 / 287$ & $0.64(0.49-0.84)$ & $0.63(0.48-0.83)$ \\
\hline \multirow[t]{2}{*}{ Statin use before ADT } & No & $2904 / 593$ & $0.58(0.46-0.73)$ & $0.67(0.53-0.84)$ \\
\hline & Yes & $1524 / 241$ & $0.68(0.51-0.91)$ & $0.88(0.66-1.18)$ \\
\hline \multirow[t]{2}{*}{ Metastatic PCa at diagnosis } & No & $3871 / 501$ & $0.75(0.62-0.90)$ & $0.74(0.61-0.90)$ \\
\hline & Yes & $556 / 333$ & $0.88(0.66-1.17)$ & $0.93(0.69-1.24)$ \\
\hline \multirow[t]{3}{*}{$\mathrm{PCa}$ risk group** } & Low risk & $768 / 72$ & $0.68(0.41-1.11)$ & $0.71(0.43-1.20)$ \\
\hline & Intermediate risk & $1639 / 150$ & $0.72(0.51-1.01)$ & $0.62(0.44-0.88)$ \\
\hline & High risk & $2021 / 612$ & $0.77(0.64-0.92)$ & $0.78(0.64-0.94)$ \\
\hline \multirow[t]{3}{*}{ Choice of primary treatment } & Active surveillance & $390 / 31$ & $0.76(0.35-1.63)$ & $0.76(0.34-1.70)$ \\
\hline & Radical prostatectomy & $332 / 60$ & $0.75(0.42-1.31)$ & $0.85(0.48-1.53)$ \\
\hline & Radical radiotherapy & $538 / 90$ & $0.74(0.48-1.16)$ & $0.74(0.46-1.18)$ \\
\hline \multirow[t]{3}{*}{ Radiation therapy } & No & $2172 / 582$ & $0.70(0.58-0.86)$ & $0.85(0.69-1.04)$ \\
\hline & Before ADT & $299 / 62$ & $0.70(0.41-1.20)$ & $0.67(0.38-1.16)$ \\
\hline & After ADT & $1957 / 190$ & $0.82(0.61-1.11)$ & $0.77(0.57-1.06)$ \\
\hline \multirow[t]{2}{*}{ Use of antidiabetic drugs } & No & $3463 / 661$ & $0.68(0.57-0.82)$ & $0.74(0.62-0.90)$ \\
\hline & Yes & $965 / 173$ & $0.68(0.50-0.94)$ & $0.74(0.53-1.02)$ \\
\hline \multirow[t]{3}{*}{ Socioeconomic status } & Employed & $503 / 119$ & $0.79(0.53-1.18)$ & $0.88(0.58-1.34)$ \\
\hline & Unemployed & $111 / 23$ & $0.53(0.15-1.83)$ & $0.77(0.21-2.86)$ \\
\hline & Retired & $3772 / 677$ & $0.66(0.56-0.79)$ & $0.71(0.60-0.85)$ \\
\hline \multirow[t]{2}{*}{ Marital status } & Single/divorced/widow & $1244 / 263$ & $0.67(0.50-0.89)$ & $0.74(0.55-1.01)$ \\
\hline & Married/registered partnership & $3184 / 571$ & $0.70(0.59-0.84)$ & $0.75(0.62-0.91)$ \\
\hline
\end{tabular}

*Calculated using Cox regression with adjustment for age, tumor risk group, randomization group, use of other medication (antidiabetic and antihypertensive drugs, NSAIDs), and whether participants received radiation therapy in addition to ADT.

**Low risk: Gleason <7, T1/2 and PSA below 10, Intermediate risk: Gleason 7, T3 or PSA between 10 and 20, High risk: Gleason >7, T4, M+ or PSA above 20.

decrease remained even after adjusting for co-medications, PCa risk group, FinRSPC randomization group and additional radiation therapy and the risk reduction was dosedependent. Especially, the finding that survival benefit was limited to statin use occurring after ADT supports synergism with ADT. This study clarifies our previous work observing increased prostate cancer survival, especially in ADT-treated patients using statins [10].

Recently, statin usage has been linked to a reduced risk of prostate cancer death, improved recurrence-free survival after radical treatment $[1,4,5]$, decreased risk of advanced prostate cancer, and decreased risk of conversion of high- 
Fig. 2 Risk of prostate cancer death by statin use after ADT stratified by subgroups. The vertical lines in figure represent $95 \%$ confidence intervals $(\mathrm{CI})$.

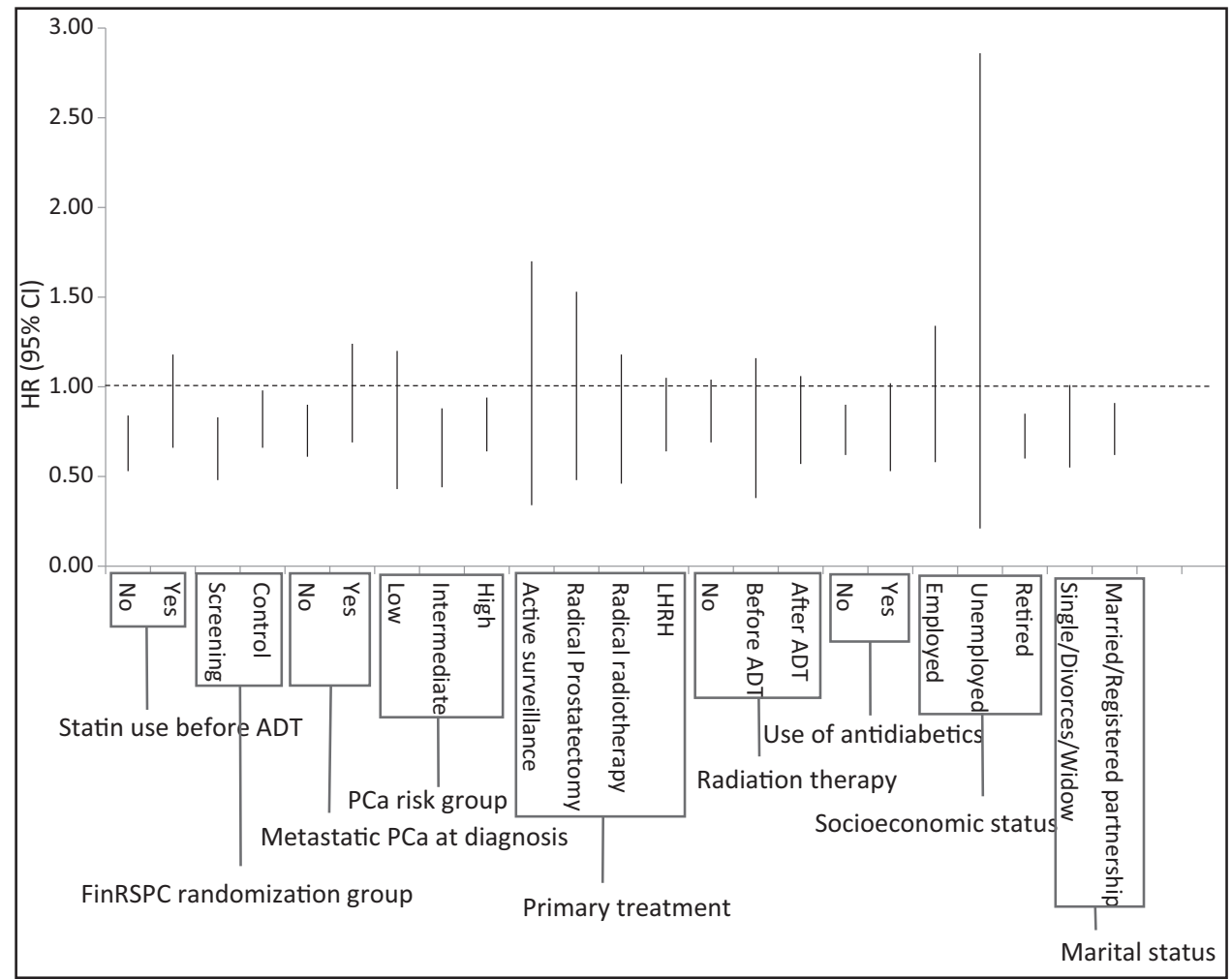

Table 5 All-cause mortality by statin use after and before ADT in a cohort of prostate cancer patients treated with ADT.

\begin{tabular}{|c|c|c|c|c|c|c|}
\hline & \multicolumn{3}{|l|}{ Statin use after ADT } & \multicolumn{3}{|c|}{ Statin use before ADT } \\
\hline & Participants/deaths & $\begin{array}{l}\text { Age-adjusted } \\
\text { HR }(95 \% \mathrm{CI})\end{array}$ & $\begin{array}{l}\text { Multivariable adjusted } \\
\text { HR }(95 \% \mathrm{CI})^{*}\end{array}$ & Participants/deaths & $\begin{array}{l}\text { Age-adjusted } \\
\text { HR }(95 \% \mathrm{CI})\end{array}$ & $\begin{array}{l}\text { Multivariable adjusted } \\
\text { HR }(95 \% \mathrm{CI})^{*}\end{array}$ \\
\hline None & $2695 / 1226$ & Reference & Reference & $2904 / 1347$ & Reference & Reference \\
\hline Any & $1733 / 701$ & $0.79(0.72-0.88)$ & $0.84(0.76-0.93)$ & $1524 / 580$ & $1.09(0.99-1.20)$ & $1.13(1.02-1.25)$ \\
\hline \multicolumn{7}{|c|}{ Amount of statin use (DDD) } \\
\hline First tertile** & $574 / 402$ & $0.81(0.66-1.00)$ & $0.84(0.68-1.04)$ & $508 / 222$ & $1.10(0.95-1.26)$ & $1.17(1.02-1.36)$ \\
\hline Second tertile & $572 / 207$ & $0.70(0.61-0.80)$ & $0.71(0.62-0.82)$ & $510 / 192$ & $1.06(0.91-1.24)$ & $1.08(0.93-1.26)$ \\
\hline Third tertile & $575 / 83$ & $0.58(0.49-0.68)$ & $0.61(0.51-0.71)$ & $506 / 166$ & $1.11(0.94-1.30)$ & $1.12(0.95-1.32)$ \\
\hline
\end{tabular}

*Calculated using Cox regression with adjustment for age, tumor risk group, randomization group, use of other medication (antidiabetic and antihypertensive drugs, NSAIDs), and whether participants received radiation therapy in addition to ADT.

**Tertiles were defined as follows for statin use after/before ADT: first tertile below 92/120 DDD/year; Second tertile 92-210/120-200; third tertile above 210/200.

grade intraepithelial neoplasia to prostate cancer [12]. Also, a recent study published by Longo et al found fluvastatin to induce PCa cell death in vitro [13]. There are few studies assessing statins' effect on ADT-treated patients [14, 15]. In these previous studies, the main results have been in line with our present study with HRs for PCa-specific mortality ranging from 0.64 to 0.76 . In this study, we were able to assess the timing of statin use in relation to the initiation of ADT. Also, statins have been associated with decreased risk of clinically significant PCa, but not overall PCa risk [16]. However, there have also been contrary results, thus uncertainty remains [17]. Considering the previous studies, we hypothesized that the beneficial effects of statins relate to cancer progression, which would explain the difference in survival. In three previous studies, statin use has been linked to better survival among ADT-treated patients $[10,18,19]$. These studies suggest that statins may enhance the therapeutic effect of ADT. In contrast, one recent Danish study [20] failed to find any differences in progression-free survival or risk of progression between statin users and non-users in PCa patients primarily treated with ADT.

Prostate cancer is known to be dependent on androgens and ADT is commonly used to manage advanced prostate 
cancer. Cholesterol, instead, is a precursor for androgen synthesis, thus it would be logical to assume that cholesterol-lowering statins target androgen synthesis. Harshman et al. proved that statins compete with androgens for influx by SLCO2B1 transporter thus inhibiting tumor's androgen supply [21]. In vitro studies have also found statins to enhance the effects of androgen-targeted drugs abiraterone acetate and enzalutamide [22]. These findings suggest that statins may improve the treatment outcomes of prostate cancer patients managed with ADT.

Epidemiologic studies assessing the association between statins and cancer survival have many potential sources of bias. Statin users may differ by socioeconomic status and health behavior from non-users. In this study, we were able to adjust for established risk factors for PCa death (TNM stage, Gleason score, PSA at diagnosis, other medications). Still, residual confounding may occur. Nevertheless, residual confounding would be expected to affect statin use similarly regardless of timing in relation to ADT. Therefore, our finding that only statin usage after ADT, not before it, was associated with improved PCa survival in a multivariable-adjusted model, supports a need for further studies to assess possible causality.

To date, only one study has assessed the effect of statins on prostate cancer survival in a randomized setting in prostate cancer patients [23]. Atorvastatin did not significantly reduce PSA or Ki-67 (a marker of cellular proliferation) overall compared to placebo, but a significant decreasing trend in tumor proliferation marker Ki- 67 by the length of atorvastatin exposure was observed. The study population consisted of men scheduled for radical prostatectomy and atorvastatin intervention was used only before surgery for a median of 21 days. Thus, the study setting differs from our study population consisting only of ADTtreated prostate cancer patients. Our current study suggests that the effect of statins on PCa may be greater in the context of ADT.

We were able to analyze statins' effect in our large population-based cohort consisting of 4428 men starting ADT. Detailed information on medication use and timing of purchases allowed us to analyze separately statin use before and after the beginning of ADT. Statins cannot be purchased over the counter in Finland. Thus, the information on statin purchases is comprehensively registered by the prescription database. Also, clinical characteristics of prostate cancer cases and causes of death were obtained from comprehensive and reliable national databases and supplemented with patient files.

We were not able to analyze the effects of physical activity, diet, and use of health services reliably as the data was missing. These unmeasured variables may have caused residual confounding. In the future, randomized clinical trials are needed to definitely evaluate the causal impact of statins on PCa survival. Further, statins' efficacy against prostate cancer may depend on the tumor's genotype, as certain PCa subtypes have been linked to increased lipid and cholesterol production [24]. We did not have information on PCa genotypes, thus we were unable to assess statins by genotype.

\section{Conclusion}

In a cohort of FinRSPC prostate cancer patients, statin use after initiation of ADT, but not before it, was associated with improved prostate cancer prognosis. This finding is in line with previous in vitro studies reporting possible synergy between statins and ADT. Randomized trials are needed to confirm or refute the survival benefit of statins among ADT-treated PCa patients.

\section{Compliance with ethical standards}

Conflict of interest TJM has served as a paid consultant for Astellas, GSK, and Janssen Cilag. TLJT has served as a paid consultant for Amgen, Astellas, Bayer, Ferring, and Roche. KT has received support from research funding from Medivation/Astellas/Pfizer, Orion, and Myovant. The remaining authors have no conflicts of interest.

Publisher's note Springer Nature remains neutral with regard to jurisdictional claims in published maps and institutional affiliations.

Open Access This article is licensed under a Creative Commons Attribution 4.0 International License, which permits use, sharing, adaptation, distribution and reproduction in any medium or format, as long as you give appropriate credit to the original author(s) and the source, provide a link to the Creative Commons license, and indicate if changes were made. The images or other third party material in this article are included in the article's Creative Commons license, unless indicated otherwise in a credit line to the material. If material is not included in the article's Creative Commons license and your intended use is not permitted by statutory regulation or exceeds the permitted use, you will need to obtain permission directly from the copyright holder. To view a copy of this license, visit http://creativecommons. org/licenses/by/4.0/.

\section{References}

1. Hamilton RJ, Banez LL, Aronson WJ, Terris MK, Platz EA, Kane $\mathrm{CJ}$, et al. Statin medication use and the risk of biochemical recurrence after radical prostatectomy: results from the shared equal access regional cancer hospital (SEARCH) database. Cancer. 2010;116:3389-98. https://doi.org/10.1002/cncr.25308

2. Metallo CM, Gameiro PA, Bell EL, Mattaini KR, Yang J, Hiller $\mathrm{K}$, et al. Reductive glutamine metabolism by IDH1 mediates lipogenesis under hypoxia. Nature. 2011;481:380-4. https://doi. org/10.1038/nature10602

3. Herber DL, Cao W, Nefedova Y, Novitskiy SV, Nagaraj S, Tyurin VA, et al. Lipid accumulation and dendritic cell dysfunction in cancer. Nat Med. 2010;16:880-6. https://doi.org/10.1038/nm. 2172

4. Allott EH, Howard LE, Cooperberg MR, Kane CJ, Aronson WJ, Terris MK, et al. Postoperative statin use and risk of biochemical 
recurrence following radical prostatectomy: results from the shared equal access regional cancer hospital (SEARCH) database. BJU Int. 2014;114:661-6. https://doi.org/10.1111/bju.12720

5. Song C, Park S, Park J, Shim M, Kim A, Jeong IG, et al. Statin use after radical prostatectomy reduces biochemical recurrence in men with prostate cancer. Prostate. 2015;75:211-7. https://doi. org/10.1002/pros. 22907

6. Larsen SB, Dehlendorff C, Skriver C, Dalton SO, Jespersen CG, Borre $\mathrm{M}$, et al. Postdiagnosis statin use and mortality in Danish patients with prostate cancer. J Clin Oncol. 2017;35:3290-7. https://doi.org/10.1200/JCO.2016.71.8981

7. Han W, Gao S, Barrett D, Ahmed M, Han D, Macoska JA. et al. Reactivation of androgen receptor-regulated lipid biosynthesis drives the progression of castration-resistant prostate cancer. Oncogene. 2018;37:710-21. https://doi.org/10.1038/onc.2017. 385.

8. Kilpeläinen TP, Tammela TL, Malila N, Hakama M, Santti H, Määttänen L, et al. Prostate cancer mortality in the Finnish randomized screening trial. J Natl Cancer Inst. 2013;105:719-25. https://doi.org/10.1093/jnci/djt038

9. Mottet N, Bellmunt J, Bolla M, Briers E, Cumberbatch MG, De Santis M. et al. EAU-ESTRO-SIOG guidelines on prostate cancer. Part 1: screening, diagnosis, and local treatment with curative intent. Eur Urol. 2017;71:618-29. https://doi.org/10.1016/j. eururo.2016.08.003.

10. Murtola TJ, Peltomaa AI, Talala K, Määttänen L, Taari K, Tammela TLJ, et al. Statin use and prostate cancer survival in the Finnish randomized study of screening for prostate cancer. Eur Urol Focus. 2017;3:212-20. https://doi.org/10.1016/j.euf.2016.05. 004

11. WHO Collaborating Centre for Drug Statistics Methodology. ATC/DDD Index 2013. 2013. http://www.whocc.no/atc_ddd_ index/

12. Longo J, Hamilton RJ, Masoomian M, Khurram N, Branchard E, Mullen PJ. et al. A pilot window-of-opportunity study of preoperative fluvastatin in localized prostate cancer. Prostate Cancer Prostatic Dis. 2020;23:630-7. https://doi.org/10.1038/s41391020-0221-7.

13. Fowke JH, Motley SS. Statin use linked with a decrease in the conversion from high-grade prostatic intraepithelial neoplasia (HGPIN) to prostate cancer. Carcinogenesis. 2018;39:819-25. https://doi.org/10.1093/carcin/bgy050

14. Wu S-Y, Fang S-C, Shih H-J, Wen Y-C, Shao Y-HJ. Mortality associated with statins in men with advanced prostate cancer treated with androgen deprivation therapy. Eur J Cancer. 2019;112:109-17. https://doi.org/10.1016/j.ejca.2018.11.032
15. Anderson-Carter I, Posielski N, Liou J-I, Khemees TA, Downs TM, Abel EJ, et al. The impact of statins in combination with androgen deprivation therapy in patients with advanced prostate cancer: a large observational study. Urol Oncol. 2019;37:130-7. https://doi.org/10.1016/j.urolonc.2018.11.017

16. Dawe DE, Ye X, Czaykowski P, Singh H, Skarsgard D, Aprikian $A$, et al. The effect of statin use on the incidence of prostate cancer: a population-based nested case-control study. Int J Cancer. 2018;143:190-8. https://doi.org/10.1002/ijc.31295

17. Jayalath VH, Nayan M, Finelli A, Komisarenki M, Timilshina N, Kulkarni GS, et al. Statin use and time to progression in men on active surveillance for prostate cancer. Prostate Cancer Prostatic Dis. 2018;21:509-15. https://doi.org/10.1038/s41391-018-0053-x

18. Harshman LC, Wang X, Nakabayashi M, Xie W, Valenca L, Werner L, et al. Statin use at the time of initiation of androgen deprivation therapy and time to progression in patients with hormone-sensitive prostate cancer. JAMA Oncol. 2015;1:495-504. https://doi.org/10.1001/jamaoncol.2015.0829

19. Yang H, Pang L, Hu X, Wang W, Xu B, Zhang X, et al. The effect of statins on advanced prostate cancer patients with androgen deprivation therapy or abiraterone/enzalutamide: a systematic review and meta-analysis. J Clin Pharm Ther. 2020;45:488-95. https://doi.org/10.1111/jcpt.13092

20. Mikkelsen MK, Thomsen FB, Berg KD, Jarden M, Larsen SB, Hansen RB, et al. Associations between statin use and progression in men with prostate cancer treated with primary androgen deprivation therapy. Scand J Urol. 2017;51:464-9. https://doi.org/ 10.1080/21681805.2017.1362032

21. Harshman LC, Werner L, Tripathi A, Wang X, Maughan BL, Antonarakis ES. et al. The impact of statin use on the efficacy of abiraterone acetate in patients with castration-resistant prostate cancer. Prostate. 2017;77:1303-11. https://doi.org/10.1002/pros.23390.

22. Syvälä H, Pennanen $P$, Bläuer M, Tammela TLJ, Murtola TJ. Additive inhibitory effects of simvastatin and enzalutamide on androgen-sensitive $\mathrm{LNCaP}$ and $\mathrm{VCaP}$ prostate cancer cells. Biochem Biophys Res Commun. 2016;481:46-50. https://doi.org/10. 1016/j.bbrc.2016.11.021

23. Murtola TJ, Syvälä H, Tolonen T, Helminen M, Riikonen J, Koskimäki J et al. Atorvastatin versus placebo for prostate cancer before radical prostatectomy - a randomized, double-blind, placebo-controlled clinical trial. Eur Urol. July 2018. https://doi. org/10.1016/j.eururo.2018.06.037

24. You S, Knudsen BS, Erho N, Alshalalfa M, Takhar M, Al-Deen Ashab $\mathrm{H}$, et al. Integrated classification of prostate cancer reveals a novel luminal subtype with poor outcome. Cancer Res. 2016;76: 4948-58. https://doi.org/10.1158/0008-5472.CAN-16-0902 